\title{
Effects of soya oligosaccharides and soya oligopeptides on lipid metabolism in hyperlipidaemic rats
}

\author{
Shali Xie ${ }^{1,2}$, Jundong Zhu ${ }^{3 *}$, Yanqi Zhang ${ }^{4}$, Kai Shi ${ }^{2}$, Yuangang $\mathrm{Shi}^{3}$ and Xiao $\mathrm{Ma}^{1 *}$ \\ ${ }^{1}$ West China School of Public Health, Sichuan University, No. 17, Section 3, South Renmin Road, Chengdu, Sichuan 610041, \\ People's Republic of China \\ ${ }^{2}$ Department of Health Education and Medical Humanities, College of Humanities and Social Sciences, Third Military \\ Medical University, No. 30 Gaotanyan Road, Chongqing 400038, People's Republic of China \\ ${ }^{3}$ Department of Nutrition and Food Hygiene, College of Military Preventive Medicine, Third Military Medical University, \\ No. 30 Gaotanyan Road, Chongqing 400038, People's Republic of China \\ ${ }^{4}$ Department of Health Statistics, College of Military Preventive Medicine, Third Military Medical University, \\ No. 30 Gaotanyan Road, Chongqing 400038, People's Republic of China
}

(Submitted 11 April 2011 - Final revision received 3 October 2011 - Accepted 8 October 2011 - First published online 15 November 2011)

\section{Abstract}

In the present study, we aimed to examine the effects of soya oligosaccharides (SOS) and soya oligopeptides (SOP) on blood lipid levels, release of vasoactive substances, antioxidant activity and faecal bile acid (FBA) excretion in rats fed a high-fat diet (HFD). Male SpragueDawley rats were evenly divided into five groups according to diets as follows: regular diet (control), HFD, HFD enriched with $2 \%$ of SOS (SOS), HFD enriched with $3 \%$ of SOP (SOP) and HFD enriched with $2 \%$ SOS and $3 \%$ SOP (SOSP). The results showed that SOS and SOP significantly reduced plasma total cholesterol, LDL-cholesterol and TAG, whereas HDL-cholesterol concentration was significantly increased. Furthermore, SOS and SOP reduced plasma apoB, apoE and the apoB:apoAI ratio, whereas apoAI was significantly increased. Moreover, SOS and SOP also reduced plasma thromboxane $\mathrm{A}_{2}\left(\mathrm{TXA}_{2}\right)$ and the $\mathrm{TXA}_{2}$ :prostacyclin $\left(\mathrm{PGI}_{2}\right)$ ratio, whereas plasma PGI 2 and nitric oxide were significantly increased. In addition, SOS and SOP significantly reduced serum and liver malondialdehyde concentrations and increased FBA excretion. However, we did not observe obvious influences of SOS and SOP on superoxide dismutase activities in the liver of HFD-fed rats. The combination of $2 \%$ SOS and $3 \%$ SOP showed a more marked effect than SOS or SOP alone in improving the lipid profile, release of vasoactive substances and increasing FBA excretion $(P<0.05)$. In summary, SOS and SOP might help prevent atherosclerosis through improving abnormal blood lipid levels, regulating vasoactive substances and protecting against oxidative stress.

\section{Key words: Soya oligosaccharides: Soya oligopeptides: Lipid metabolism: Atherosclerosis}

CVD is the leading cause of mortality in the world, accounting for the deaths of seventeen million people each year or approximately one-third (29\%) of global deaths annually ${ }^{(1)}$. CVD prevention is a global public health priority. According to the estimation of the WHO, $88 \%$ of the global mortality and disease burden from CVD occurs in low- and middleincome countries ${ }^{(1)}$. For example, CVD causes over three million deaths each year in China, and at least 230 million Chinese are suffering from CVD. The origin of CVD is multifactorial and its pathological basis is atherosclerosis. Hypercholesterolaemia is recognised as a major and independent risk factor of atherosclerosis and $\mathrm{CVD}^{(2)}$. About $60-70 \%$ of plasma cholesterol is present in the LDL fraction ${ }^{(3)}$. Plasma LDL-cholesterol (LDL-C) crosses the endothelial barrier and reaches the vascular subendothelial intima, whereby promoting monocyte/macrophage differentiation. Subsequently, monocyte-derived macrophages take up cholesterol through scavenger receptors and become lipid-laden foam cells ${ }^{(4)}$. Steinberg et al. $^{(5)}$ showed that the cholesterol accumulating in macrophage-derived foam cells comes from circulating lipoproteins, mainly from the atherogenic LDL. Therefore, plasma lipids play a critical role in the initiation and progression of the atherosclerotic lesion ${ }^{(4)}$, and it is essential to keep blood cholesterol levels in the appropriate range for

Abbreviations: AI, atherosclerosis index; BW, body weight; FBA, faecal bile acid; HDL-C, HDL-cholesterol; HFD, high-fat diet; LDL-C, LDL-cholesterol; MDA, malondialdehyde; $\mathrm{PGI}_{2}$, prostacyclin; SOD, superoxide dismutase; SOP, soya oligopeptides; SOS, soya oligosaccharides; SOSP, HFD enriched with $2 \%$ SOS and $3 \%$ SOP; TC, total cholesterol; TXA , thromboxane $\mathrm{A}_{2}$.

*Corresponding authors: Professor J. Zhu, email zjdnfs@126.com; Professor X. Ma, fax +86 285585080 , email huaxihe@gmail.com 
the prevention of atherosclerosis and CVD. On the basis of a meta-analysis, a $1 \%$ decrease in plasma cholesterol level can reduce the risk of CVD up to $3 \%^{(6)}$.

Hypolipidaemic drugs, particularly statins, are the preferred treatment for reducing blood cholesterol levels because of their efficacy and safety ${ }^{(7)}$. However, dietary modification is the mainstay of treatment of lipid abnormalities and can have a significant cholesterol-lowering effect ${ }^{(8)}$.

Soyabean, as a traditional Chinese food, has been consumed for thousands of years in China due to its beneficial effects on health. Soya contains a variety of phytochemicals with special health functions, such as soya isoflavones, saponins, phytic acid, sterol and so on. Besides these, soya oligosaccharides (SOS) and soya oligopeptides (SOP) are two types of bioactive substances extracted from soyabean. SOS is the general term for soluble sugars in soyabean, and it is mainly composed of stachyose, raffinose and sucrose. SOP refers to peptides with molecular weights lower than $1000 \mathrm{Da}$, and it is derived from soya protein by enzyme hydrolysis. Some reports ${ }^{(9,10)}$ have suggested that both soya protein and SOP have effects on improving the lipid profile in hypercholesterolaemic subjects, whereas SOP is more effective than soya protein. Many studies found that oligosaccharides have cholesterol-lowering properties. However, little information is known about the effect of SOS on lipoprotein metabolism in high-cholesterol-fed animals, especially the combined effects of SOS and SOP on improving dyslipidaemia. It has been shown that probiotics, such as bifidobacteria and lactobacilli, have beneficial effects on cholesterol metabolism in vitro and in vivo ${ }^{(11)}$. Lan et al. ${ }^{(12)}$ reported that SOS is a powerful prebiotic that can increase caecal bifidobacterium and lactobacilli populations in animals and human subjects. SOP is the necessary and suitable nutrient for the growth of intestinal bifidobacteria ${ }^{(13)}$

In the present study, we aimed to determine whether SOS and/or SOP had significant effects on lipid metabolism in a rat model with hyperlipidaemia induced by a high-fat diet (HFD). Besides plasma lipid parameters, we examined vasoactive substances, antioxidant activity and faecal bile acid (FBA) excretion in order to explore the potential application of SOS and SOP in CVD prevention. The second aim of the study was to determine whether the combination of SOS and SOP had additive or synergistic effects on these parameters.

\section{Materials and methods}

\section{Materials}

SOS extracted from soyabean whey was provided by Shanghai Far-reaching Food Company Limited (Shanghai, China). The total sugar content was $\geq 70 \%$.

SOP was provided by Shandong Zhongshi Duqing Biotech Company Limited (Jinan, China). The peptide content was $\geq 80 \%$.

\section{Animals, diets and experimental protocol}

A total of sixty healthy male Sprague-Dawley rats were obtained from the Laboratory Animal Center of the Third
Military Medical University, and the body weight (BW) ranged from 190 to $210 \mathrm{~g}$. The animals were individually housed in metabolic cages in an environment-controlled room $\left(20-23^{\circ} \mathrm{C}\right.$ and $40-60 \%$ of humidity) under a $12 \mathrm{~h}$ light $-12 \mathrm{~h}$ dark cycle with free access to food and water. All animals were fed for 8 weeks, and BW was monitored biweekly throughout the study. Food intake was measured three times per week. The animal protocol was approved by the Third Military Medical University Institutional Animal Care Committee and conducted in accordance with the Third Military Medical University guidelines for the care and use of laboratory animals.

After a week of adaptation, sixty Sprague-Dawley rats were evenly divided into five groups according to diets as follows: regular diet (control), HFD, HFD enriched with $2 \%$ of SOS (SOS), HFD enriched with $3 \%$ of SOP (SOP) and HFD enriched with $2 \%$ SOS and 3\% SOP (SOSP). The regular diet was composed of the following ingredients $(\mathrm{g} / 100 \mathrm{~g}$ diet): bean cake, 20; wheat starch, 19; maize starch, 15; rice starch, 20; bran, 15; fishmeal, 5; bone meal, 3; yeast, 1; salt, 1 ; and cod liver oil, 1 . The total protein/carbohydrate/fat content of the regular diet was approximately 18, 52 and 5\%, respectively. The ingredients of the HFD were as follows: $10 \%$ lard, $2 \%$ cholesterol, $1 \%$ bile salt and $87 \%$ regular diet. In the SOS group, HFD was supplemented with $2 \%$ SOS by replacing $2 \mathrm{~g}$ of bran. In the SOP group, HFD was supplemented with $3 \%$ SOP by replacing $3 \mathrm{~g}$ of fishmeal. In the SOSP group, HFD was supplemented with $2 \%$ SOS and $3 \%$ SOP by replacing $2 \mathrm{~g}$ of bran and $3 \mathrm{~g}$ of fishmeal, respectively. Diets of the HFD and experimental groups were iso-energetic and iso-nitrogenic. At the end of the experiment, after overnight fasting, rats were anaesthetised using pentobarbital sodium, and blood samples from the femoral vein were collected in tubes containing EDTA $(1.4 \mathrm{~g} / \mathrm{l})$ and Trasylol $(100 \mathrm{kU} / 1)$. Then, plasma was separated for subsequent biochemical analysis and stored at $-70^{\circ} \mathrm{C}$. Immediately after killing the animal, liver tissues were dissected and stored at $-70^{\circ} \mathrm{C}$ for later antioxidant activity assay. Atherosclerosis index $(\mathrm{AI})$ was calculated according to the Fridewald formula:

$$
\mathrm{AI}=\mathrm{LDL}-\mathrm{C} / \mathrm{HDL}-\mathrm{C} \text {. }
$$

All faeces were collected for three consecutive days at the end of the experiment. The collected faeces were stored at $-20^{\circ} \mathrm{C}$ for later determination of FBA content by phosphomolybdate colorimetry after $16 \mathrm{~h}$ of vacuum freeze-drying.

\section{Biochemical analysis}

Serum lipid profile analysis. Serum total cholesterol (TC), TAG, LDL-C and HDL-cholesterol (HDL-C) levels were determined by commercial enzymatic test kits (Biosino Bio-technology and Science Inc., Beijing, China) using an automatic biochemical analyser (Type AU2700; Olympus, Tokyo, Japan). ApoAI, apoB and apoE were determined by immunoturbidimetry using assay kits from Biosino Biotechnology and Science Inc. These procedures were fully automated and have been described in detail elsewhere ${ }^{(14)}$. 
Evaluation of antioxidant activities. Superoxide dismutase (SOD) activity in the serum and liver was determined by the xanthine oxidase method using a commercially available kit (SOD Detection Kit; Nanjing Jiancheng Bioengineering Institute, Nanjing, China) ${ }^{(15)}$. Malondialdehyde (MDA) in the serum and liver was measured by the thiobarbituric acid method $^{(16)}$ using a commercially available kit (MDA Detection Kit, Nanjing Jiancheng Bioengineering Institute). The assay was based on the ability of a chromogenic agent to react with MDA, yielding a stable chromophore with maximal absorbance at $532 \mathrm{~nm}$.

Prostanoid release measurements. In order to measure the release of thromboxane $\mathrm{A}_{2}\left(\mathrm{TXA}_{2}\right)$ and prostacyclin $\left(\mathrm{PGI}_{2}\right)$, direct RIA was carried out to determine the concentrations of thromboxane $\mathrm{B}_{2}$ and $6-\mathrm{ketoPGF}_{1 \mathrm{a}}$, the stable degradation products of $\mathrm{TXA}_{2}$ and $\mathrm{PGI}_{2}$, respectively ${ }^{(17)}$. RIA kits were supplied by the General Hospital of PLA, Beijing, China.

Plasma nitric oxide assay. Plasma NO concentration was determined by colorimetric technique and nitrate reductase method using a Nitrate/Nitrite Colorimetric Assay kit (Nanjing Jiancheng Bioengineering Institute). This assay was based on the enzymatic conversion of nitrate to nitrite by nitrate reductase, and the concentration of $\mathrm{NO}$ was indirectly measured by determining both nitrate and nitrite levels in the plasma.

Determination of faecal bile acids. Total FBA was determined by spectrophotometry based on the colour reaction between phosphomolybdate and bile acid ${ }^{(18)}$. Protein and pigment were precipitated from faeces by adding ethanol, and total bile acids were extracted by chloroform and methanol $(2: 1, \mathrm{v} / \mathrm{v})$. Phosphomolybdate reagent (phosphomolybdate $(2.5 \mathrm{~g})$, glacial acetic acid $(100 \mathrm{ml})$ and concentrated sulphuric acid $(10 \mathrm{ml})$ ) was used to form colour reaction, and then it was quantified by a 721 spectrophotometer (wavelength: $690 \mathrm{~nm}$ ) (Shanghai Phenix Optical Scientific Instrument Company Limited, Shanghai, China).

\section{Statistical analysis}

All statistical calculations were carried out with Statistical Package of SPSS 13.0 (Release 13.01S China: Beijing Stats Data Mining Company Limited, Beijing, China; permanent license). Data were expressed as mean values and standard deviations. For
Table 1. Effects of soya oligosaccharides (SOS) and soya oligopeptides (SOP) on body weight and food intake of rats* (Mean values and standard deviations, $n 12$ per group)

\begin{tabular}{|c|c|c|c|c|c|c|c|c|}
\hline \multirow[b]{2}{*}{ Group } & \multicolumn{2}{|c|}{$\begin{array}{c}\text { Initial } \\
\text { weight }(\mathrm{g})\end{array}$} & \multicolumn{2}{|c|}{$\begin{array}{c}\text { Final } \\
\text { weight (g) }\end{array}$} & \multicolumn{2}{|c|}{$\begin{array}{l}\text { Weight } \\
\text { gain }(\mathrm{g})\end{array}$} & \multicolumn{2}{|c|}{$\begin{array}{c}\text { Food } \\
\text { intake }(g / d)\end{array}$} \\
\hline & Mean & SD & Mean & SD & Mean & SD & Mean & SD \\
\hline Control & 240 & 16 & 320 & 21 & 78 & 16 & $15 \cdot 4$ & 4.3 \\
\hline HFD & 237 & 15 & 368 & 23 & 129 & 27 & 17.5 & 4.0 \\
\hline sos & 238 & 19 & 363 & 25 & 122 & 24 & $19 \cdot 1$ & 6.5 \\
\hline SOP & 239 & 16 & 373 & 27 & 130 & 20 & 17.9 & $5 \cdot 0$ \\
\hline SOSP & 238 & 19 & 374 & 25 & 131 & 20 & $18 \cdot 7$ & 4.5 \\
\hline
\end{tabular}

HFD, high-fat diet; SOSP, HFD enriched with $2 \%$ SOS and $3 \%$ SOP.

${ }^{*}$ There were no significant difference between groups.

data with normal distribution and homogeneity of variance, significance of difference $(P<0 \cdot 05)$ between mean values was determined by ANOVA coupled with the least significant differences (LSD) test, whereas it was determined by the Dunnett-T3 test for data with heterogeneity of variance.

\section{Results}

\section{Body weight and food intake}

Table 1 shows that neither the pre-treatment BW of rats nor weight gains after the 8-week treatment were significantly different among the five groups. The mean daily food intake of rats in the HFD and experimental groups was higher than that of the control group during the 8-week experiment; however, the difference was not statistically significant.

\section{Lipid profile changes}

Table 2 shows the effects of SOS and SOP on serum TC, TAG, LDL-C and HDL-C levels. Serum TC, TAG and LDL-C levels in the HFD group were significantly higher than those of the control group, whereas HDL-C level was significantly lower than that of the control group $(P<0.05)$. The addition of SOS and SOP alone or together with HFD resulted in significantly decreased serum TC, TAG and LDL-C levels, whereas HDL-C level was significantly increased compared with the HFD group $(P<0.05)$. In particular, the combination of SOS

Table 2. Effects of soya oligosaccharides (SOS) and soya oligopeptides (SOP) on lipid profile of rats (Mean values and standard deviations, $n 12$ per group)

\begin{tabular}{|c|c|c|c|c|c|c|c|c|}
\hline \multirow[b]{2}{*}{ Group } & \multicolumn{2}{|c|}{$\mathrm{TC}(\mathrm{mmol} / \mathrm{l})$} & \multicolumn{2}{|c|}{ TAG (mmol/l) } & \multicolumn{2}{|c|}{ LDL-C (mmol/l) } & \multicolumn{2}{|c|}{ HDL-C (mmol/l) } \\
\hline & Mean & SD & Mean & SD & Mean & SD & Mean & SD \\
\hline Control & 2.59 & 0.25 & 0.29 & 0.02 & 1.03 & 0.23 & 1.50 & 0.13 \\
\hline HFD & $6 \cdot 26^{*}$ & 0.52 & $2 \cdot 76^{*}$ & 0.28 & $4 \cdot 68^{*}$ & 0.35 & $1.04^{*}$ & 0.13 \\
\hline sos & $3.90 * \dagger$ & 0.34 & $1.37^{\star} \dagger$ & 0.14 & $1.90^{*} \dagger$ & 0.27 & $2 \cdot 28^{*} \dagger$ & 0.13 \\
\hline SOP & $4.09^{*} \dagger$ & 0.23 & $1 \cdot 65^{\star} \dagger \ddagger$ & 0.08 & $1.55^{*} \dagger \ddagger$ & 0.24 & $2 \cdot 21^{*} \dagger$ & 0.11 \\
\hline SOSP & $3 \cdot 50^{\star} \dagger \neq \S$ & 0.19 & $1 \cdot 31^{*}+\S$ & 0.07 & $1.07 \dagger+\S$ & 0.22 & $2 \cdot 42^{\star} \dagger \neq \S$ & 0.10 \\
\hline
\end{tabular}

TC, total cholesterol; LDL-C, LDL-cholesterol; HDL-C, HDL-cholesterol; HFD, high-fat diet; SOSP, HFD enriched with $2 \%$ SOS and $3 \%$ SOP.

${ }^{*}$ Mean values were significantly different from those of the control group $(P<0.05)$.

† Mean values were significantly different from those of the HFD group $(P<0.05)$.

$\ddagger$ Mean values were significantly different from those of the SOS group $(P<0.05)$.

$\S$ Mean values were significantly different from those of the SOP group $(P<0.05)$. 


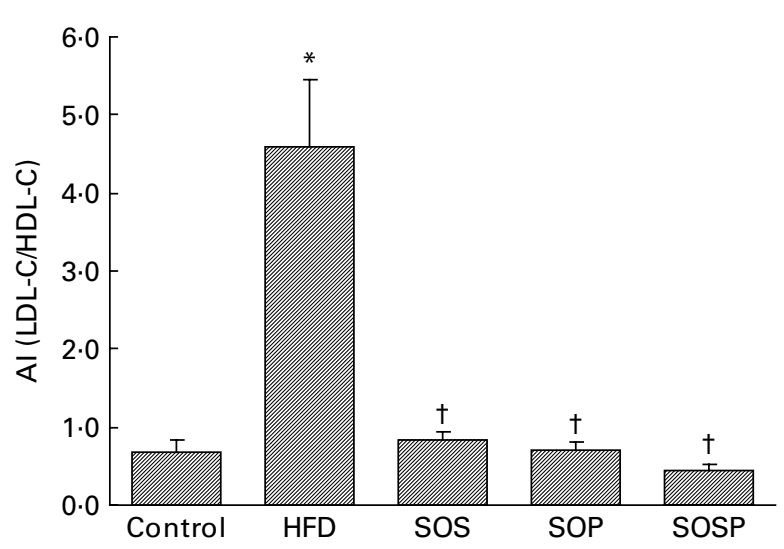

Fig. 1. Changes of atherosclerosis index (Al). Values are means and standard deviations represented by vertical bars. * Mean value was significantly different from that of the control group $(P<0.05)$. † Mean values were significantly different from that of the high-fat diet (HFD) group $(P<0.05)$. LDL-C, LDL-cholesterol; HDL-C, HDL-cholesterol; SOP, soya oligopeptides; SOS, soya oligosaccharides; SOSP, HFD enriched with $2 \%$ SOS and $3 \%$ SOP.

and SOP was significantly more effective than SOS and SOP alone in altering TC, LDL-C and HDL-C levels $(P<0.05)$.

Fig. 1 shows the change of AI value of each group. AI value of the HFD group was significantly higher than that of the control group, whereas the value of the three experimental groups was significantly lower than that of the HFD group $(P<0 \cdot 05)$. There was no statistically significant difference among the control and experimental groups.

\section{Effects of soya oligosaccharides and soya oligopeptides on plasma apo levels}

Table 3 shows the effects of SOS and SOP on plasma apo levels in rats. Plasma apoAI level in the HFD group was significantly lower, whereas apoB and apoE levels were significantly higher than those of the control group $(P<0 \cdot 05)$. ApoAI levels of the three experimental groups were significantly increased compared with the HFD and control groups $(P<0 \cdot 05)$, and the most significant effect was observed in the SOSP group $(P<0.05)$. ApoB levels of the SOP and SOSP groups were significantly decreased compared with those of the other three groups, and apoB level of the SOS group was significantly decreased compared with that of the HFD group $(P<0.05)$.
ApoE level of the three experimental groups was significantly lower than that of the HFD group $(P<0.05)$, and the values were not statistically different from the control except for the SOS group. Comparisons within the three experimental groups revealed that the significant difference was only obtained between the SOS and SOSP groups $(P<0 \cdot 05)$.

In terms of the apoB:apoAI ratio, it was significantly increased in the HFD group compared with that of the control group, whereas it was significantly decreased in the three experimental groups compared with that in both HFD and control groups $(P<0 \cdot 05)$. Furthermore, the apoB:apoAI ratio of the three experimental groups was in an order as follows: SOSP group $<$ SOP group $<$ SOS group $(P<0.05)$.

\section{Effects of soya oligosaccharides and soya oligopeptides on vasoactive substances}

Table 4 shows the effects of SOS and SOP on plasma vasoactive substances. Plasma $\mathrm{PGI}_{2}$ and NO levels of the HFD group were significantly lower than those of the control group, whereas $\mathrm{TXA}_{2}$ and the TXA $2: \mathrm{PGI}_{2}$ ratio of the HFD group were significantly higher than those of the control group $(P<0 \cdot 05)$. Plasma $\mathrm{PGI}_{2}$ and NO levels of the three experimental groups were significantly higher than those of the HFD group, whereas $\mathrm{TXA}_{2}$ and the TXA 2 : $\mathrm{PGI}_{2}$ ratio of the three experimental groups were significantly lower than those of the HFD group, but higher than those of the control group $(P<0 \cdot 05)$. Compared within the three experimental groups, the SOSP group was more effective in reducing $\mathrm{TXA}_{2}$ and the $\mathrm{TXA}_{2}: \mathrm{PGI}_{2}$ ratio, as well as increasing NO than the SOS or SOP group $(P<0 \cdot 05)$.

\section{Superoxide dismutase activity and malondialdehyde content in livers and serum}

Table 5 shows that SOD activity in the serum, as well as MDA contents in the liver and serum of the HFD group were significantly higher than those of the control group $(P<0 \cdot 05)$, whereas SOD activity in the liver of the HFD group was not statistically different from that of the control group. SOD activity in the liver of the three experimental groups was significantly lower than that of the control group $(P<0.05)$. SOD activity in serum and the MDA content in the liver and serum of the

Table 3. Effects of soya oligosaccharides (SOS) and soya oligopeptides (SOP) on plasma apo levels of rats

(Mean values and standard deviations, $n 12$ per group)

\begin{tabular}{|c|c|c|c|c|c|c|c|c|}
\hline \multirow[b]{2}{*}{ Group } & \multicolumn{2}{|c|}{ ApoAI (g/l) } & \multicolumn{2}{|c|}{ ApoB (g/l) } & \multicolumn{2}{|c|}{ ApoE (mg/l) } & \multicolumn{2}{|c|}{ ApoB:apoAl } \\
\hline & Mean & SD & Mean & $\mathrm{SD}$ & Mean & $\mathrm{SD}$ & Mean & $\mathrm{SD}$ \\
\hline Control & 0.81 & 0.02 & 0.66 & 0.15 & $37 \cdot 1$ & $2 \cdot 6$ & 0.82 & 0.18 \\
\hline HFD & $0.45^{\star}$ & 0.03 & $0.90^{*}$ & 0.03 & $46 \cdot 8^{*}$ & 3.7 & $1.99^{\star}$ & 0.19 \\
\hline sos & $1.34^{*} \dagger$ & 0.02 & $0.51 \dagger$ & 0.04 & $33 \cdot 1^{*} \dagger$ & 3.0 & $0.38^{*} \dagger$ & 0.04 \\
\hline SOP & $1.42^{\star} \dagger \ddagger$ & 0.08 & $0.40^{*} \dagger \ddagger$ & 0.02 & $35.9 \dagger$ & $2 \cdot 6$ & $0.28^{\star} \dagger \ddagger$ & 0.01 \\
\hline SOSP & $1.51^{*} \dagger \ddagger \S$ & 0.06 & $0.35^{\star} \dagger \ddagger$ & 0.03 & 36.9†‡ & $2 \cdot 0$ & $0.23^{\star} \dagger \ddagger \S$ & 0.02 \\
\hline
\end{tabular}

HFD, high-fat diet; SOSP, HFD enriched with $2 \%$ SOS and $3 \%$ SOP.

${ }^{*}$ Mean values were significantly different from those of the control group $(P<0.05)$.

† Mean values were significantly different from those of the HFD group $(P<0.05)$.

$\ddagger$ Mean values were significantly different from those of the SOS group $(P<0.05)$.

$\S$ Mean values were significantly different from those of the SOP group $(P<0.05)$. 
Table 4. Effects of soya oligosaccharides (SOS) and soya oligopeptides (SOP) on vasoactive substances of rats (Mean values and standard deviations, $n 12$ per group)

\begin{tabular}{|c|c|c|c|c|c|c|c|c|}
\hline \multirow[b]{2}{*}{ Group } & \multicolumn{2}{|c|}{$\mathrm{TXA}_{2}(\mathrm{ng} / \mathrm{l})$} & \multicolumn{2}{|c|}{$\mathrm{PGI}_{2}(\mathrm{ng} / \mathrm{l})$} & \multicolumn{2}{|c|}{$\mathrm{NO}(\mu \mathrm{mol} / \mathrm{l})$} & \multicolumn{2}{|c|}{$\mathrm{TXA}_{2}: \mathrm{PGI}_{2}$} \\
\hline & Mean & SD & Mean & SD & Mean & $\mathrm{SD}$ & Mean & SD \\
\hline Control & 97 & 8 & 136 & 5 & 35.4 & $2 \cdot 1$ & 0.72 & 0.05 \\
\hline HFD & $511^{*}$ & 27 & $86^{*}$ & 9 & $23 \cdot 8^{\star}$ & $2 \cdot 0$ & $6.01^{*}$ & 0.77 \\
\hline sos & $209^{*} \dagger$ & 18 & $158^{*} \dagger$ & 11 & $37.3 \dagger$ & $2 \cdot 2$ & $1.33^{*} \dagger$ & 0.12 \\
\hline SOP & $237^{\star} \dagger \ddagger$ & 23 & $144 \dagger \ddagger$ & 9 & $35.4 \dagger$ & 2.0 & $1.65^{\star} \dagger \ddagger$ & 0.16 \\
\hline SOSP & $186^{*} \dagger \ddagger \S$ & 10 & $146^{*} \dagger \ddagger$ & 10 & $38 \cdot 6^{*}+\S$ & 2.4 & $1.28^{*}+\S$ & 0.12 \\
\hline
\end{tabular}

$\mathrm{TXA}_{2}$, thromboxane $\mathrm{A}_{2} ; \mathrm{PGI}_{2}$, prostacyclin; NO, nitric oxide; HFD, high-fat diet; SOSP, HFD enriched with $2 \%$ SOS and $3 \%$ SOP.

* Mean values were significantly different from those of the control group $(P<0.05)$.

$\dagger$ Mean values were significantly different from those of the HFD group $(P<0.05)$.

$\ddagger$ Mean values were significantly different from those of the SOS group $(P<0.05)$.

$\S$ Mean values were significantly different from those of the SOP group $(P<0.05)$.

three experimental groups were significantly higher than those of the control group, but lower than those of the HFD group $(P<0.05)$, except for the liver MDA content of the SOSP group, which was not significantly different from that of the control group. Among the three experimental groups, the MDA content in the liver of the SOSP group was significantly lower than that of the SOS group $(P<0 \cdot 05)$, whereas no significance was obtained from the other experimental groups.

\section{Dry faecal weight and bile acid excretion}

Table 6 shows the FBA levels of rats. Dry faecal weight of the HFD group and the three experimental groups were higher than that of the control group. However, there were no significant differences among any groups $(P>0 \cdot 05)$. FBA of the three experimental groups was significantly higher than that of the HFD and control groups, and FBA of the HFD group was significantly higher than that of the control group $(P<0 \cdot 05)$. Compared with the three experimental groups, FBA content of the SOSP group was significantly higher than that of the other two experimental groups $(P<0 \cdot 05)$.

\section{Discussion}

In the present study, we did not observe significant differences of the mean daily food intake and BW gains among five groups, indicating that the consumption of diet by rats was not altered by high fat, SOS and SOP, and thus the BW gains were not significantly different among the five groups.

The reduction of TC or LDL-C in plasma has been reported to reduce the risk of CVD. Numerous studies in human subjects and animals have shown the hypocholesterolaemic effect of soya protein ${ }^{(10,19,20)}$. Some studies reported that the effect of soya protein on lipid metabolism is attributed to its component of peptides ${ }^{(21,22)}$. In the present study, we observed that serum TC and TAG of the SOP group were decreased by 34.7 and $40 \cdot 2 \%$ compared with the HFD group, respectively. These results are consistent with findings of Duranti et al. ${ }^{(22)}$, who showed that plasma TC and TAG of rats fed a hypercholesterolaemic diet were reduced by 36 and 34\%, respectively, by treatment with $20 \mathrm{mg} / \mathrm{kg}$ BW per d of soya $7 \mathrm{~S}$ globulin- $\alpha$ ' subunit for $28 \mathrm{~d}$. By suppressing cholesterol micelle solubility, Zhong et al. ${ }^{(23)}$ showed that SOP interferes with the micellar solubilisation of cholesterol, resulting in decreased intestinal cholesterol absorption and ensuing serum cholesterol reduction.

Chen et $_{\text {al }}{ }^{(24)}$ reported that SOS effectively reduces serum TC and TAG levels, thus improving the HFD-induced abnormal blood lipid level in rats. Similarly, the present study showed that SOS reduced serum TC and TAG concentrations of hyperlipidaemic rats by 37.7 and $50 \cdot 4 \%$, respectively. With a powerful prebiotic ability, SOS is fermented in the colon by bifidobacterium, yielding large amounts of SCFA, such as butyrate and

Table 5. Effects of soya oligosaccharides (SOS) and soya oligopeptides (SOP) on superoxide dismutase (SOD) activity and malondialdehyde (MDA) content in liver and serum of rats

(Mean values and standard deviations, $n 12$ per group)

\begin{tabular}{|c|c|c|c|c|c|c|c|c|}
\hline \multirow[b]{3}{*}{ Group } & \multicolumn{4}{|c|}{ SOD } & \multicolumn{4}{|c|}{ MDA } \\
\hline & \multicolumn{2}{|c|}{$\begin{array}{c}\text { Livers } \\
\text { (U/mg protein) }\end{array}$} & \multicolumn{2}{|c|}{ Serum $(\mathrm{U} / \mathrm{ml})$} & \multicolumn{2}{|c|}{$\begin{array}{c}\text { Livers } \\
\text { (nmol/g liver) }\end{array}$} & \multicolumn{2}{|c|}{ Serum (nmol/l) } \\
\hline & Mean & SD & Mean & SD & Mean & SD & Mean & SD \\
\hline Control & 662 & 26 & 346 & 16 & $12 \cdot 8$ & 1.9 & 5.98 & 0.21 \\
\hline HFD & 598 & 60 & $738^{*}$ & 69 & $29 \cdot 6^{*}$ & 4.5 & $16 \cdot 52^{*}$ & 0.99 \\
\hline sos & $581^{*}$ & 41 & $424^{\star} \dagger$ & 41 & $23 \cdot 6^{*} \dagger$ & $4 \cdot 2$ & $8 \cdot 47^{\star} \dagger$ & 0.72 \\
\hline SOP & $565^{\star}$ & 100 & $428^{*} \dagger$ & 53 & $19 \cdot 9^{*} \dagger$ & $8 \cdot 6$ & $7.99^{*} \dagger$ & 0.52 \\
\hline SOSP & $572^{\star}$ & 35 & $423^{*} \dagger$ & 51 & $15 \cdot 2 \dagger \ddagger$ & $4 \cdot 2$ & $7 \cdot 84^{*} \dagger$ & 0.48 \\
\hline
\end{tabular}

HFD, high-fat diet; SOSP, HFD enriched with $2 \%$ SOS and $3 \%$ SOP.

${ }^{*}$ Mean values were significantly different from those of the control group $(P<0.05)$.

† Mean values were significantly different from those of the HFD group $(P<0.05)$.

$\ddagger$ Mean values were significantly different from those of the SOS group $(P<0.05)$. 
Table 6. Effects of soya oligosaccharides (SOS) and soya oligopeptides (SOP) on faecal dry weight and bile acids of rats

(Mean values and standard deviations, $n 12$ per group)

\begin{tabular}{|c|c|c|c|c|}
\hline \multirow[b]{2}{*}{ Group } & \multicolumn{2}{|c|}{$\begin{array}{l}\text { Dry weight } \\
\text { (g/3d) }\end{array}$} & \multicolumn{2}{|c|}{$\begin{array}{c}\text { Bile acids } \\
\text { ( } \mu \mathrm{g} / \mathrm{g} \text { dry weight })\end{array}$} \\
\hline & Mean & $S D$ & Mean & SD \\
\hline Control & 13.2 & 3.1 & 48 & 8 \\
\hline HFD & $14 \cdot 6$ & 1.9 & $126^{*}$ & 20 \\
\hline sos & 15.5 & $2 \cdot 4$ & $167^{\star} \dagger$ & 7 \\
\hline SOP & $15 \cdot 1$ & 4.0 & $151^{*} \dagger$ & 13 \\
\hline SOSP & $17 \cdot 2$ & 4.1 & $196^{*} \dagger \ddagger \S$ & 16 \\
\hline
\end{tabular}

HFD, high-fat diet; SOSP, HFD enriched with $2 \%$ SOS and $3 \%$ SOP.

${ }^{*}$ Mean values were significantly different from those of the control group $(P<0.05)$. † Mean values were significantly different from those of the HFD group $(P<0.05)$.

$\ddagger$ Mean values were significantly different from those of the SOS group $(P<0.05)$.

$\S$ Mean values were significantly different from those of the SOP group $(P<0.05)$.

propionate $^{(25)}$. It has been hypothesised that SCFA, in particular propionate and butyrate, are able to influence lipid metabolism $^{(26)}$. Butyrate can inhibit hepatic cholesterol synthesis, while propionate may inhibit the synthesis of fatty acids in the liver, thereby reducing the rates of TAG secretion ${ }^{(27)}$. Therefore, the present data suggested that the improved lipid metabolism observed in the SOS group was partly caused by an alteration in the absorption and/or synthesis of cholesterol through SCFA. In the present study, we observed that the combined treatment of SOS and SOP reduced TC and TAG levels by $10.3,14.4$ and $4.4 \%, 20.6 \%$ compared with SOS and SOP alone, respectively, suggesting that the combination of SOS and SOP was more effective in improving dyslipidaemia, thus reducing the risk of atherosclerotic CVD.

There is increasing evidence that both reducing LDL-C and raising HDL-C can result in significant cardiovascular benefit ${ }^{(28)}$. In the present study, LDL-C was reduced by $66.9 \%$ in the SOP group and by $59.4 \%$ in the SOS group, whereas HDL-C was increased by 112.5 and $119 \cdot 2 \%$, respectively. These results suggested that these effects of SOS and SOP on LDL-C and HDL-C were beneficial for atherosclerotic CVD. Some studies confirmed the reduction in plasma TC and LDLC without significant effect on HDL-C by soya protein ${ }^{(19,29)}$. Zhao et al. ${ }^{(30)}$ observed that SOP reduces serum TC and TAG levels of hyperlipidaemic rats, whereas HDL-C is not significantly altered. However, we observed a significant increase of plasma HDL-C in the SOP group. The precise reason for this inconsistency with other reports remains unclear. It may result from the SOP used in the present study, which was different from other studies, especially the molecular weight of SOP. It has been proposed that the regulation of cholesterol homoeostasis by SOP depends on the activation of LDL receptors and LDL degradation in the liver ${ }^{(31)}$. Lovati et al. ${ }^{(32)}$ demonstrated that peptides derived from soya 7 S globulin- $\alpha$ ' subunit are able to up-regulate LDL receptors' activity, thus resulting in receptor-mediated $\mathrm{LDL}$ catabolism ${ }^{(33)}$. However, $7 \mathrm{~S}$ lacking the $\alpha$-subunit has no effect on the up-regulation of LDL receptors, indicating that soya peptides with hypocholesterolaemic activity are involved in the regulation of LDL receptors. The observed decrease in AI values suggested that SOS and SOP, especially their combination, were auxiliary in decreasing the risk of atherosclerosis and CVD.

High apoB, the structural protein of LDL, and a high apoB:apoAI ratio are strongly related to the increased atherosclerotic cardiovascular risk. In contrast, high apoAI, mainly representing HDL and being crucial in transferring cholesterol from tissues to the liver, is inversely related to atherogenic risk $^{(34)}$. In the present study, we observed a significantly higher apoB and apoB:apoAI ratio, exhibiting an atherogenic lipid profile as well as a significantly lower apoAI in HFD-fed rats. The present data also showed a significant reduction of apoB by $43.3 \%$ and an increase of apoAI by $197.8 \%$ in SOSfed rats. In a clinical study, Teixeira et al. ${ }^{(20)}$ observed that soya protein effectively reduces plasma apoB, whereas the change in apoAI is not significant. However, in the present study, SOP not only reduced apoB but also increased apoAI effectively. Moreover, the combination of SOP and SOS showed a more marked effect on reducing the apoB:apoAI ratio and increasing apoAI than SOP and SOS alone. These data indicated that the combination of SOP and SOS could be more effective in reducing serum lipid risk factors for CVD than SOP or SOS alone.

Endothelial dysfunction plays an important role in the early development of atherosclerosis ${ }^{(35)} \cdot \mathrm{PGI}_{2}$ and $\mathrm{NO}$ are antiatherogenic because of their vasodilating effects, whereas $\mathrm{TXA}_{2}$ is a potent vasoconstrictor ${ }^{(36-38)}$. In the present study, we observed that HFD reduced the release of $\mathrm{PGI}_{2}$ and $\mathrm{NO}$, and increased the $\mathrm{TXA}_{2}$ level, suggesting that endotheliumdependent vasorelaxation was attenuated by hyperlipidae$\mathrm{mia}^{(39)}$, and the effect of hypercholesterolaemia on endothelial cells leads to the inhibition of NO synthesis ${ }^{(35)}$. In vitro study has shown that SOP increases the release of $\mathrm{PGI}_{2}$ and $\mathrm{NO}$, but it does not affect $\mathrm{TXA}_{2}{ }^{(40)}$. The present study showed that SOP not only increased the release of $\mathrm{PGI}_{2}$ and $\mathrm{NO}$ by 67.9 and $48.9 \%$, respectively, but also decreased $\mathrm{TXA}_{2}$ by $53.6 \%$ compared with the HFD group. SOS exerted similar effects. Furthermore, the combination of SOS and SOP was more effective in reducing $\mathrm{TXA}_{2}$ and the $\mathrm{TXA}_{2}: \mathrm{PGI}_{2}$ ratio, as well as increasing NO than SOS or SOP alone, thus improving endothelial dysfunction and cardiovascular health.

It has been shown that oxidative stress contributes to endothelial dysfunction and atherosclerosis ${ }^{(2)}$. However, dietary antioxidants may protect against oxidative events in the body, and the antioxidant activity reduces CVD by preventing LDL oxidation ${ }^{(35,41)}$. In the present study, oxidative stress occurred in HFD-fed rats with elevated liver and serum levels of MDA, the ultimate product of lipid peroxidation. The antioxidant defence system, such as SOD, is important to protect the body from oxidative damage ${ }^{(42)}$. Many experimental results showed that HFD or hyperlipidaemia can reduce SOD activity in rats ${ }^{(43)}$. Surprisingly, in the present study, the serum SOD activity of the HFD group was not reduced, and it was higher than that of the control and experimental groups. The present findings are consistent with those of Arafa et al. ${ }^{(44)}$, who showed that a HFD does not decrease the liver SOD activity, which is higher than that of normal dietfed rats and curcumin-treated rats. We supposed that the result was due to the high level of MDA, which resulted in the 
increase of SOD activity for maintaining the balance between oxidative stress and antioxidant defences.

Gibbs et al. ${ }^{(45)}$ reported that the antioxidant activity of soya protein is associated with the fractions $11 \mathrm{~S}$ or glycinin as the precursor of antioxidant peptides. In another study, it has been shown that the ingestion of soya peptide in rats reduces paraquat-induced oxidative stress by preventing the elevation of the concentration of serum thiobarbituric acid-reactive substances, thus effectively preventing LDL oxidation ${ }^{(46)}$. The present data show that SOP decreases MDA by 32.8 and $51.6 \%$ in the liver and serum, respectively, thus reducing oxidative stress. Chen et al. ${ }^{(47)}$ found that peptides with a ProHis-His sequence demonstrate the greatest antioxidant activity among all tested peptides, and their activity is decreased by removing a His residue from the C-terminus. Chen et al. ${ }^{(24)}$ reported that the activities of antioxidant enzymes in the liver and serum, such as catalase and SOD, are significantly elevated when SOS is orally administered to rats for $45 \mathrm{~d}$. The present data show that SOS could reduce oxidative stress by increasing the serum SOD activity and reducing the MDA content in SOS-treated rats, suggesting that SOS protected blood vessels and endothelial cells from oxidative damage by inhibiting LDL oxidation, thus protecting against atherosclerosis.

Bile acids are critical in maintaining cholesterol homoeostasis. Binding of bile acids and increasing their faecal excretion have been hypothesised as an effective way to reduce cholesterol by dietary factors ${ }^{(48)}$. Iwami et al. ${ }^{(49)}$ reported that SOP binds bile acids, thereby enhancing bile acid and faecal steroid excretion, which may contribute to the hypocholesterolaemic activity. In the present study, SOP-fed rats demonstrated significantly greater FBA excretion than those fed with normal diets or HFD. Greater FBA excretion leads to the increased bile acid production from cholesterol, thus reducing serum cholesterol concentrations. Choi et al. ${ }^{(50)}$ identified the bile acid-binding region in SOP and found that the glycininderived peptide VAWWMY is responsible for this bile acidbinding ability. Therefore, the present data support the hypothesis that the absorption of cholesterol and bile acids could be further decreased by SOP, and the increased bile acid excretion is an important contributor to the hypocholesterolaemic effect of SOP. SOS-fed rats also showed significantly higher excretion of FBA compared with the control and HFD groups. A $30 \mathrm{~d}$ study in human subjects ${ }^{(25)}$ showed that SOS intake of $3 \mathrm{~g} / \mathrm{d}$ significantly increases faecal bifidobacteria counts, SCFA concentrations and lipid output in women. The cholesterol-lowering effect of bifidobacteria is attributed to increasing deconjugation of bile acids, production of SCFA and cholesterol binding in the intestinal lumen $^{(11)}$. Therefore, we suggested that increasing FBA excretion associated with promotion of intestinal bifidobacteria proliferation and the production of SCFA by SOS is also important for blood cholesterol-lowering and cardioprotective effects. In the present study, we observed that a combination of SOS and SOP was more effective in increasing FBA excretion than SOS and SOP alone. SOS is a powerful prebiotic oligosaccharide, while SOP is also suitable to sustain growth of intestinal bifidobacteria. Therefore, the increasing
FBA excretion by the combination of SOS and SOP could be ascribed to an additive or synergistic effect.

In summary, the present study shows that ingestion of $2 \%$ SOS or 3\% SOP influence lipid metabolism, release of vasoactive substances as well as antioxidant activity and increase FBA excretion. In particular, the combination of $2 \%$ SOS and $3 \%$ SOP is more effective than SOS or SOP alone, especially in improving the lipid profile and increasing FBA excretion. These results indicate that SOS and SOP have potential value in CVD prevention. Further studies should focus on clarifying the mechanisms of action of the lipid effects of SOS and SOP.

\section{Acknowledgements}

The authors thank the Department of Nutrition and Food Hygiene, Third Military Medical University, for laboratory and technical support of the present study. They are also grateful to Dr Fang Chen and Junhua Peng for their valuable discussions on the methods of bile acid measurement. This work was supported by the Sci \& Tech Research Project Foundation of Scientific Committee of Chongqing (200300168). S. X., J. Z. and X. M. designed the research; S. X. conducted the research; Y. Z., K. S. and Y. S. analysed the data; and S. X. and J. Z. wrote the manuscript.

\section{References}

1. World Health Organization (2008) The Global Burden of Disease: 2004 Update. Geneva, Switzerland: WHO. http:// www.who.int/healthinfo/global_burden_disease/GBD_report_ 2004update_full.pdf (accessed September 2010).

2. Lusis AJ (2000) Atherosclerosis. Nature 407, 233-241.

3. Halpern MJ (1995) Lipids and atherosclerosis. Mol Aspects Med 16, 509-710.

4. Rader DJ \& Puré E (2005) Lipoproteins, macrophage function, and atherosclerosis: beyond the foam cell? Cell Metab 1, 223-230.

5. Steinberg D, Parthasarathy S, Carew TE, et al. (1989) Beyond cholesterol. Modifications of low-density lipoprotein that increase its atherogenicity. $N$ Engl J Med 320, 915-924.

6. Brown BG, Stukovsky KH \& Zhao XQ (2006) Simultaneous lowdensity lipoprotein-C lowering and high-density lipoprotein-C elevation for optimum cardiovascular disease prevention with various drug classes, and their combinations: a meta-analysis of 23 randomized lipid trials. Curr Opin Lipidol 17, 631-636.

7. Armitage J (2007) The safety of statins in clinical practice. Lancet 370, 1781-1790.

8. Bruckert E \& Rosenbaum D (2011) Lowering LDL-cholesterol through diet: potential role in the statin era. Curr Opin Lipidol 22, 43-48.

9. Sugano M, Goto S, Yamada Y, et al. (1990) Cholesterollowering activity of various undigested fractions of soybean protein in rats. $J$ Nutr 120, 977-985.

10. Anderson JW, Johnstone BM \& Cook-Newell ME (1995) A meta-analysis of the effects of soy protein intake on serum lipids. N Engl J Med 333, 276-282.

11. Taylor GR \& Williams CM (1998) Effects of probiotics and prebiotics on blood lipids. Br J Nutr 80, S225-S230.

12. Lan Y, Williams BA, Verstegena MWA, et al. (2007) Soy oligosaccharides in vitro fermentation characteristics and its effect on caecal microorganisms of young broiler chickens. Anim Feed Sci Technol 133, 286-297. 
13. Bezkorovainy A (2001) Probiotics: determinants of survival and growth in the gut. Am J Clin Nutr 73, 399S-405S.

14. Ikeda T, Shibuya Y, Senba U, et al. (1991) Automated immunoturbidimetric analysis of six plasma apoliproteins: correlation with radial immunodiffusion assays. J Clin Lab Anal 5, 90-95.

15. Oyanagui Y (1984) Reevaluation of assay methods and establishment of kit for superoxide dismutase activity. Anal Biochem 142, 290-296.

16. Chen CY, Huang YL \& Lin TH (1998) Association between oxidative stress and cytokine production in nickel-treated rats. Arch Biochem Biophys 356, 127-132.

17. Bouaziz A, Marie M, Richert A, et al. (1998) Direct physical factors and $\mathrm{PGI}_{2}$ and $\mathrm{TXA}_{2}$ secretions by a human endothelial cell line: in vitro investigation of pressure and shear stress applied independently or in synergy. Thromb Res 90, 279-289.

18. Hong NK \& Meng CW (1983) Determination of total bile acids in bile by phosphomolybdate colorimetry. Shanghai Med J 6, 35-37.

19. Tonstad S, Smerud K \& Høie L (2002) A comparison of the effects of 2 doses of soy protein or casein on serum lipids, serum lipoproteins, and plasma total homocysteine in hypercholesterolemic subjects. Am J Clin Nutr 76, 78-84.

20. Teixeira SR, Potter SM, Weigel R, et al. (2000) Effects of feeding 4 levels of soy protein for 3 and $6 \mathrm{wk}$ on blood lipids and apolipoproteins in moderately hypercholesterolemic men. Am J Clin Nutr 71, 1077-1084.

21. Hori G, Wang MF, Chan YC, et al. (2001) Soy protein hydrolyzate with bound phospholipids reduces serum cholesterol levels in hypercholesterolemic adult male volunteers. Biosci Biotechnol Biochem 65, 72-78.

22. Duranti M, Lovati MR, Dani V, et al. (2004) The alpha' subunit from soybean $7 \mathrm{~S}$ globulin lowers plasma lipids and upregulates liver beta-VLDL receptors in rats fed a hypercholesterolemic diet. J Nutr 134, 1334-1339.

23. Zhong F, Zhang XM, Ma JG, et al. (2007) Fractionation and identification of a novel hypocholesterolemic peptide derived from soy protein Alcalase hydrolysates. Food Res Int 40, 756-762.

24. Chen H, Liu LJ, Zhu JJ, et al. (2010) Effect of soybean oligosaccharides on blood lipid, glucose levels and antioxidant enzymes activity in high fat rats. Food Chem 119, 1633-1636.

25. Bang MH, Chio OS \& Kim WK (2007) Soy oligosaccharide increases fecal bifidobacteria counts, short-chain fatty acids, and fecal lipid concentrations in young Korean women. J Med Food 10, 366-370.

26. Bornet F, Alamowitch C \& Slama G (1994) Short chain fatty acids and metabolic effects in humans. In Gum and Stabilisers for Food Industry, 7th ed., pp. 217-229 [GO Philips, DJ Weldlock and TE Williams, editors]. Oxford: Oxford University Press.

27. Trautwein EA, Rieckhoff D \& Erbersdobler HF (1998) Dietary inulin lowers plasma cholesterol and triacylglycerol and alters biliary bile acid profile in hamsters. J Nutr 128, 1937-1943.

28. Sirtori CR \& Fumagalli R (2006) LDL-cholesterol lowering or HDL-cholesterol raising for cardiovascular prevention. A lesson from cholesterol turnover studies and others. Atherosclerosis 186, 1-11.

29. Hermansen K, Sondergaard M, Høie L, et al. (2001) Beneficial effects of a soy-based dietary supplement on lipid levels and cardiovascular risk markers in type 2 diabetic subjects. Diabetes Care 24, 228-233.

30. Zhao XJ, Wang XX, Wu BX, et al. (2002) Regulation effects of soy active peptides on lipid parameters of rats fed a high fat diet. Chin J Health Lab Technol 12, 421-422.
31. Erdmann K, Cheung BW \& Schröder H (2008) The possible roles of food-derived bioactive peptides in reducing the risk of cardiovascular disease. J Nutr Biochem 19, 643-654.

32. Lovati MR, Manzoni C, Gianazza E, et al. (2000) Soy protein peptides regulate cholesterol homeostasis in Hep G2 cells. J Nutr 130, 2543-2549.

33. Manzoni C, Duranti M, Eberini I, et al. (2003) Subcellular localization of soybean $7 \mathrm{~S}$ globulin in HepG2 cells and LDL receptor up-regulation by its alphaV constituent subunit. J Nutr 133, 2149-2155.

34. Walldius G, Jungner I, Aastveit AH, et al. (2004) The apoB/ apoA-I ratio is better than the cholesterol ratios to estimate the balance between plasma proatherogenic and antiatherogenic lipoproteins and to predict coronary risk. Clin Chem Lab Med 42, 1355-1363.

35. Sitia S, Tomasoni L, Atzeni F, et al. (2010) From endothelial dysfunction to atherosclerosis. Autoimmun Rev 9, 830-834.

36. Praticò D, Cyrus T, Li H, et al. (2000) Endogenous biosynthesis of thromboxane and prostacyclin in 2 distinct murine models of atherosclerosis. Blood 96, 3823-3826.

37. Naseem KM (2005) The role of nitric oxide in cardiovascular diseases. Mol Aspects Med 26, 33-65.

38. Var A, Yildirim Y, Onur E, et al. (2003) Endothelial dysfunction in preeclampsia. Increased homocysteine and decreased nitric oxide levels. Gynecol Obstet Invest 56, 221-224.

39. Dusting GJ, Hyland R, Hickey H, et al. (1995) Angiotensinconverting enzyme inhibitors reduce neointimal thickening and maintain endothelial nitric oxide function in rabbit carotid arteries. Am J Cardiol 76, 24E-27E.

40. Ringseis R, Matthes B, Lehmann V, et al. (2005) Peptides and hydrolysates from casein and soy protein modulate the release of vasoactive substances from human aortic endothelial cells. Biochim Biophys Acta 1721, 89-97.

41. Mesa MD, Silván JM, Olza J, et al. (2008) Antioxidant properties of soy protein-fructooligosaccharide glycation systems and its hydrolyzates. Food Res Int 41, 606-615.

42. Mahfouz MM \& Kummerow FA (2000) Cholesterol-rich diets have different effects on lipid peroxidation, cholesterol oxides, and antioxidant enzymes in rats and rabbits. $J$ Nutr Biochem 11, 293-302.

43. Watanabe S \& Fukui T (2000) Suppressive effect of curcumin on trichloroethylene-induced oxidative stress. J Nutr Sci Vitaminol (Tokyo) 46, 230-234.

44. Arafa HM (2005) Curcumin attenuates diet-induced hypercholesterolemia in rats. Med Sci Monit 11, BR228-BR234.

45. Gibbs BF, Zougman A, Masse R, et al. (2004) Production and characterization of bioactive peptides from soy hydrolysate and soy-fermented food. Food Res Int 37, 123-131.

46. Takenaka A, Annaka H, Kimura Y, et al. (2003) Reduction of paraquat-induced oxidative stress in rats by dietary soy peptide. Biosci Biotechnol Biochem 67, 278-283.

47. Chen HM, Muramoto K, Yamauchi F, et al. (1996) Antioxidant activity of designed peptides based on the antioxidative peptide isolated from digests of a soybean protein. J Agric Food Chem 44, 2619-2623.

48. Anderson JW \& Siesel AE (1990) Hypocholesterolemic effects of oat products. In New Developments In Dietary Fiber: Physiological, Physiochemical, and Analytical Aspects, pp. 17-36 [I Furda and CJ Brine, editors]. New York, NY: Plenum Press.

49. Iwami K, Sakakibara K \& Ibuki F (1986) Involvement of postdigestion hydrophobic peptides in plasma cholesterol-lowering effect of dietary plant protein. Agric Biol Chem 50, 1217-1222.

50. Choi SK, Adachi M \& Utsumi S (2002) Identification of the bile acid-binding region in the soy glycinin A1aB1b subunit. Biosci Biotechnol Biochem 66, 2395-2401. 